\title{
Relación entre tipos sociométricos y autoatri- bución académica del fracaso en una muestra de españoles de Educación Secundaria
}

\section{Cándido J. Inglés ${ }^{1}$, D. Aparisi ${ }^{2}$, B. Delgado ${ }^{2}$, L. Granados ${ }^{2}$ y José M. García-Fernández ${ }^{2}$ \\ ${ }^{1}$ Departamento de Psicología de la Salud, Universidad Miguel Hernández de Elche, Alicante \\ ${ }^{2}$ Departamento de Psicología Evolutiva y Didáctica, Universidad de Alicante, Alicante}

\section{España}

Correspondencia: D. Aparisi. Universidad de Alicante. Avenida de Carretera San Vicente s/n. 03080 (Alicante). España. E-mail: david.aparisi@ua.es

(C) Education \& Psychology I+D+i and Ilustre Colegio Oficial de la Psicología de Andalucía Oriental (Spain) 


\section{Resumen}

Introducción. El objetivo de este estudio fue analizar la relación entre tipos sociométricos, categorías conductuales y la autoatribución académica del fracaso (a la Capacidad, al Esfuerzo o a Causas Externas), en Lectura, en Matemáticas y en General.

Método. La muestra fue 1349 adolescentes españoles de 12 a 16 años. La identificación sociométrica de los estudiantes se realizó mediante el Programa Socio y para el análisis de las autoatribuciones académicas se administró la Escala de Atribución de Sydney (Sydney Attribution Scale (SAS).

Resultados. Los resultados muestran que los estudiantes nominados negativamente por sus compañeros obtuvieron puntuaciones significativamente más altas en la autoatribución del fracaso en Lectura, Matemáticas y en General a la Capacidad y al Esfuerzo que los estudiantes nominados positivamente por sus compañeros.

Discusión y conclusiones. Los tipos sociométricos resultaron un predictor significativo de las autoatribuciones académicas del fracaso, ya que los estudiantes nominados negativamente por sus compañeros presentaron mayor probabilidad de atribuir el fracaso académico a causas internas como la Capacidad y el Esfuerzo que los nominados positivamente.

Palabras Clave: adolescencia, tipos sociométricos, autoatribuciones académicas, educación secundaria. 


\begin{abstract}
Introduction. The aim of this study was to analyze the relationship between sociometric types, behavioral categories and academic self-attribution of failure (Capacity, Effort or External Causes) in Reading, Mathematics and General.

Method. A total sample of 1349 Spanish adolescents 12-16 year participed. Sociometric student identification was performed by the Programa Socio and for the analysis of academic self-attributions Sydney Attribution Scale (SAS; Marsh, 1984) was administered.

Results. Results show that students nominated by their peers negatively obtained significantly higher scores on the self-attribution of failure to the Capacity and Effort in Reading, Mathematics and General than students positively nominated by their peers.

Discussion and Conclusion. Sociometric types were a significant predictor of academic failure self-attributions as negatively students nominated by their peers were more likely to attribute academic failure to internal causes as the Capacity and Effort that positively nominees.
\end{abstract}

Keywords: adolescence, sociometric types, academic self-attributions, secondary education. 


\section{Introducción}

Es innegable la importancia del grupo de pares en el proceso de socialización (Coronel, Levin y Mejail, 2011). En este sentido, el ingreso a la escuela secundaria marca la aparición de nuevas reglas, nuevos modos y nuevos espacios de encuentro (Díaz-Aguado, 2005).

El estatus sociométrico se considera a menudo como un reflejo de la competencia social de un niño o adolescente entendida como la capacidad de éstos para implicarse con éxito en interacciones, relaciones y grupos (Rubin, Bukowski y Parker, 2006). En este sentido, las técnicas sociométricas proporcionan la posibilidad de evaluar en poco tiempo y con gran validez las relaciones entre compañeros, permitiendo obtener información del nivel de adaptación de cada sujeto y de los contextos en los que se desarrolla (Martínez-Arias, Martín y DíazAguado, 2009). Inglés, Delgado, García-Fernández, Ruiz-Esteban y Díaz-Herrero (2010) analizaron la relación entre los estilos de interacción social (agresividad, prosociabilidad y ansiedad social) y los tipos sociométricos (preferido, rechazado e ignorado). Los resultados revelaron que los estudiantes prosociales fueron proporcionalmente más elegidos por sus compañeros como preferidos, los estudiantes agresivos fueron los más rechazados por sus compañeros y los estudiantes identificados con ansiedad social fueron elegidos como los menos preferidos entre sus compañeros y, además, resultaron ser más rechazados e ignorados que los prosociales.

La educación secundaria es una etapa importante en el ciclo vital de todos los estudiantes, pues los adolescentes han de decidir si desean proseguir con sus estudios o reorientar su camino hacia el mundo laboral. En este sentido, conocer las causas de las atribuciones académicas de los estudiantes en esta etapa puede resultar beneficioso para orientarles en la elección de su futuro profesional (Bain y Allin, 2005).

\section{Tipos sociométricos y autoatribuciones académicas}

La popularidad y el estatus sociométrico en el grupo de iguales se ha estudiado en relación con el ajuste psicosocial (Garaigordobil, 2006), el ajuste escolar (Martín, 2011) y las atribuciones académicas. En este sentido, la mayoría de las investigaciones se centran en la forma en que los estudiantes interpretan el rechazo que pueden estar sufriendo y las atribuciones de causalidad que realizan sobre su situación. Por ejemplo, Muñoz, Trianes y Jiménez (1994) plantean que el rechazo social (en términos de un bajo estatus sociométrico) origina un 
autoconcepto social negativo que el sujeto atribuye a causas internas, estables e incontrolables por su parte (falta de habilidades, de capacidad, o antipatía). Este tipo de atribución genera futuras expectativas de fracaso en las relaciones con los demás con su consiguiente repercusión en los resultados escolares.

Jiménez (2003) examinó las diferencias en variables auto y hetero-evaluadas de distintos grupos sociométricos (populares, rechazados, controvertidos e intermedios) desde una perspectiva múltiple en una muestra de 443 estudiantes de primaria, los cuales, rellenaron cuestionarios sociométricos, de autoconcepto, de locus de control y de ansiedad-evitación social. Los resultados mostraron que los niños populares fueron caracterizados por un patrón de conducta positiva y prosocial, en tanto que los rechazados por una conducta negativa y agresiva-perturbadora. Respecto a las atribuciones sobre las relaciones interpersonales, no hubo diferencias entre los grupos en internalidad y controlabilidad para los éxitos y fracasos sociales. Como se esperaba, los rechazados puntuaron más bajo que los otros grupos, pero no encontraron diferencias estadísticamente significativas.

La investigación de Zhao y Su (2005) se centró en el estudio de las interpretaciones de las situaciones de rechazo social de 376 adolescentes. Concluyeron que el modo en que los adolescentes rechazados por sus iguales utilizan para interpretar el comportamiento de otras personas está relacionado con el grado de aceptación social, es decir, realizan atribuciones diferentes en el caso de compararse con otros estudiantes también rechazados o con los estudiantes populares.

Si nos centramos en las autoatribuciones académicas, cabe señalar que los patrones o estilos atribucionales básicos de alumnos de Educación Secundaria en relación con el rendimiento académico son un exponente y un indicativo muy importante a la hora de buscar una explicación causal al proceso de estudio, aprendizaje y rendimiento de los alumnos. Intervienen varios factores, por una parte la motivación hacia el alto rendimiento o éxito escolar se mantiene o incrementa cuando los sujetos atribuyen su éxito o buen rendimiento académico a factores internos (capacidad y esfuerzo) y sobre todo estables (capacidad), aunque también, y en menor medida, a factores inestables (esfuerzo). El hecho importante a destacar aquí es que este tipo de atribuciones produce en el sujeto unos sentimientos de autoconfianza, autoestima, de valoración positiva y de satisfacción hacia su propia persona, lo que influye directamente 
en su autoconcepto y su propia competencia social. Todo ello redunda y determina un efecto positivo sobre la motivación de rendimiento y la motivación de aprendizaje (Hayamizu y Weiner, 1991).

El estudio llevado a cabo en 2002 por Jiayan Pan analizó la relación entre el estatus social, personalidad y estilos de atribución causal en una muestra de 527 estudiantes de secundaria de China. Los resultados mostraron cómo el estilo atribucional y los rasgos de personalidad influían en el estatus social de los estudiantes de secundaria concluyendo que los estudiantes rechazados muestran diferencias significativas en la atribución de los éxitos académicos a causas externas.

\section{El presente estudio}

Aunque la evidencia empírica previa ha puesto de manifiesto la existencia de cierto paralelismo entre diversas variables cognitivo-motivacionales implicadas en los dominios académico y social (por ejemplo, las autoatribuciones académicas y el estatus social), de tal forma que, podría afirmarse que en general, el éxito o fracaso en el contexto académico tiende a covariar con el éxito o fracaso en el contexto social y viceversa (Chen, Chang y He, 2003), existe una carencia de trabajos que examinen concretamente la relación entre las autoatribuciones académicas y los tipos sociométricos en adolescentes españoles que están cursando la Educación Secundaria.

Por tanto, este trabajo pretende aportar nuevos datos a la investigación sobre la relación existente entre tipos sociométricos y las autoatribuciones académicas en estudiantes españoles, ampliando el número de tipos sociométricos examinados (populares, rechazadosagresivos, rechazados-tímidos e ignorados-olvidados) y categorías conductuales que pueden aparecer dentro de un grupo-aula (líder, simpático, colaborador, peleón, obediente y buen estudiante).

\section{Objetivos e hipótesis}

Concretamente, el presente estudio tiene como objetivos específicos: a) analizar las diferencias en las autoatribuciones académicas del fracaso (Capacidad y Esfuerzo) en Lectura, Matemáticas y General entre adolescentes españoles en función de los tipos sociométricos y las categorías conductuales anteriormente indicadas, y b) comprobar si las autoatribuciones 
académicas son una variable predictora y estadísticamente significativa de los tipos sociométricos y categorías conductuales.

A partir de la evidencia empírica previa, se plantean las siguientes hipótesis: 1) los estudiantes nominados negativamente por sus iguales (rechazados-agresivos, rechazadostímidos, olvidados, peleones y obedientes) presentarán puntuaciones significativamente superiores en la autoatribución de sus fracasos a causas internas (Capacidad y Esfuerzo) en Lectura, Matemáticas y General a partir de la Escala de Atribución de Sydney, que los estudiantes nominados positivamente por sus iguales (populares, líderes, simpáticos, colaboradores y buenos estudiantes), y 2) los tipos sociométricos y categorías conductuales serán una variable predictora estadísticamente significativa de las autoatribuciones académicas del fracaso en la presente muestra.

\section{Método}

\section{Participantes}

Se realizó un muestreo aleatorio por conglomerados (zonas geográficas de la Región de Murcia y la provincia de Alicante: centro, norte, sur, este y oeste). Con el fin de que todas las zonas geográficas estuvieran representadas, se seleccionaron aleatoriamente entre 1 y 3 centros por zona en función de la población, computándose en total 20 centros de áreas rurales y urbanas (14 públicos y 6 privados). Una vez determinados los centros del estudio, se seleccionaron aleatoriamente cuatro aulas computándose aproximadamente 120 sujetos por centro.

El total de sujetos reclutados fue 1594 estudiantes de $1^{\circ}$ a $4^{\circ}$ de ESO (error muestral $=$ $.02)$, de los que $76(4.77 \%)$ fueron excluidos por errores u omisiones en sus respuestas, 40 (2.51\%) por no obtener por escrito el consentimiento informado de los padres para participar en la investigación y 129 (8.09\%) fueron excluidos por ser extranjeros con importantes déficit en el dominio de la lengua española. Por tanto, la muestra definitiva se compuso de 1349 estudiantes (697 chicos y 652 chicas), con un rango de edad de 12 a 16 años $(M=13.81 ; D T=$ 1.35). El $86.30 \%$ de los estudiantes no estaba repitiendo curso. La composición étnica de la muestra fue la siguiente: $88.9 \%$ españoles, $6.34 \%$ hispanoamericanos, $3.37 \%$ resto de Europa, $.75 \%$ asiáticos y $.64 \%$ árabes. La distribución de los sujetos por sexo y curso académico fue la siguiente: 386 en $1^{\circ}$ de ESO (203 chicos y 183 chicas), 325 en $2^{\circ}$ de ESO (173 chicos y 152 
chicas), 318 en $3^{\circ}$ de ESO (172 chicos y 146 chicas) y 320 en $4^{\circ}$ de ESO (149 chicos y 171 chicas). Por medio de la prueba Chi-cuadrado de homogeneidad de la distribución de frecuencias, se comprobó que no existían diferencias estadísticamente significativas entre los ocho grupos de sexo x curso $\left(\chi^{2}=4.53 ; p=.21\right)$.

\section{Instrumentos}

Test de Nominación Sociométrica. El test sociométrico es un instrumento que permite descubrir las formas de interacción de los individuos dentro de los grupos y revelar la estructura del grupo con el fin de identificar personas preferidas, rechazadas e ignoradas, así como figuras líderes, cooperativas, conflictivas, etc. El método sociométrico de nominación se basa en la medición de la atracción y la repulsión hacia los miembros de un grupo (Moreno, 1934), identificadas a partir de las elecciones y rechazos informados por los estudiantes, y se clasifican mediante las dimensiones de preferencia social e impacto social, propuestas por Peery (1979). Teniendo en cuenta estas dos dimensiones ortogonales y mediante técnicas estadísticas los sujetos pueden ser identificados como preferidos, rechazados, ignorados, controvertidos y medios.

Este trabajo se centró en el análisis de sujetos preferidos-populares, rechazados (rechazados-agresivos y rechazados-tímidos) e ignorados-olvidados, ya que son éstos los que agrupan el mayor número de alumnos y, a su vez, representan el mejor (preferidos) y peor ajuste social (rechazado e ignorados) en el contexto académico (García-Bacete, 2007). La identificación sociométrica de los estudiantes se realizó mediante el Programa Socio (González, 1990) que permite obtener los límites inferiores y superiores de las nominaciones positivas recibidas y de las nominaciones negativas recibidas para un grupo o clase de alumnos.

Además, se analizaron las distintas categorías conductuales que pueden aparecer dentro de un grupo social: líder, simpático, colaborador, peleón, obediente-sumiso y buen estudiante. Se empleó el procedimiento de nominación probabilística de tres elecciones intergénero, considerado como el más adecuado y ajustado en pruebas de nominación sociométrica (García-Bacete, 2007).

Escala de Atribución de Sydney (Sydney Attribution Scale, SAS; Marsh, 1984; adaptado por Núñez y González-Pienda, 1994). Es una escala multidimensional que mide las per- 
cepciones de los estudiantes acerca de las causas del sus éxitos y fracasos académicos. Está diseñada a partir de 24 situaciones hipotéticas donde los estudiantes deben responder sobre una escala Likert de 5 puntos (Falso $=1$, Verdadero $=5$ ). La escala resulta de la combinación de tres dimensiones: (a) dos áreas académicas (Verbal-lectura, Matemática); (b) dos resultados hipotéticos (Éxito, Fracaso) y; (c) tres tipos de causas (Capacidad, Esfuerzo, Causas Externas). La combinación de las 24 situaciones (seis para el éxito y otras seis para el fracaso para cada área académica) referidas a tres posibles causas genera un total de 72 ítems. El cuestionario se sustenta en la teoría atribucional de Weiner $(1986,2004)$, la cual postula que los sujetos ante un resultado, ya sea positivo (éxito) o negativo (fracaso), tienden a explicarlo a través de causas o factores causales como la capacidad (o falta de capacidad), el esfuerzo (o falta de esfuerzo), el azar o suerte y la dificultad o facilidad de la tarea. Estas causas están caracterizadas por las dimensiones de locus de control (interno al sujeto vs. externo al sujeto), estabilidad (estable en el tiempo vs. variable en el tiempo) y controlabilidad (el sujeto cree que controla la causa vs. el sujeto no cree controlar la causa). De este modo, la capacidad es considerada una causa interna, estable e incontrolable, el esfuerzo es una causa interna, inestable y controlable, la suerte es una causa externa, inestable e incontrolable y la dificultad de la tarea es una causa externa, estable e incontrolable. Las propiedades psicométricas de la SAS, halladas a partir de muestras de estudiantes australianos, chilenos, españoles, norteamericanos y filipinos indican que esta escala constituye una medida excelente para evaluar autoatribuciones académicas en Educación Primaria y Secundaria. También han sido constatadas sus propiedades psicométricas adecuadas en población universitaria (Inglés, Rodríguez-Marín y González-Pienda, 2008).

\section{Procedimiento}

Se llevó a cabo una entrevista con los directores y orientadores de los centros participantes para exponer los objetivos de la investigación, describir los instrumentos de evaluación, solicitar permiso y promover su colaboración. Posteriormente, se celebró una reunión con los padres para explicarles el estudio y solicitar el consentimiento informado por escrito autorizando a sus hijos a participar en la investigación.

Los cuestionarios fueron contestados de forma colectiva y voluntaria en el aula, asignando previamente un número de identificación a las hojas de respuesta entregadas a cada sujeto, las cuales fueron posteriormente corregidas mediante ordenador. A continuación, se 
leyeron en voz alta las instrucciones, enfatizando la importancia de no dejar ninguna pregunta sin contestar. Los investigadores estuvieron presentes durante la administración de las pruebas para aclarar posibles dudas y verificar la administración independiente por parte de los participantes.

\section{Análisis de datos}

La identificación sociométrica de los estudiantes se realizó mediante el Programa Socio (González, 1990) que permite obtener los límites inferiores y superiores de las nominaciones positivas recibidas (LI (Np) y LS $(\mathrm{Np})$ ) y de las nominaciones negativas recibidas (LI (Nn) y LS (Nn)) para un grupo de alumnos. Estos límites se obtienen a través de los cálculos de la probabilidad binomial, con el fin de encontrar el valor de la prueba $t$ asociado a una asimetría determinada y un nivel de probabilidad $<.05$ (tablas de Salvosa). La identificación se consigue aplicando los siguientes criterios: Preferidos $=\mathrm{Np} \geq \mathrm{LS}(\mathrm{Np})$ y $\mathrm{Nn}<\mathrm{M}(\mathrm{Nn})$, Rechazados $=\mathrm{Nn} \geq \mathrm{LS}(\mathrm{Nn})$ y $\mathrm{Np}<\mathrm{M}(\mathrm{Np})$, e Ignorados $=\mathrm{Np} \leq 1$ y $\mathrm{Nn}<\mathrm{M}(\mathrm{Nn})$.

Con el objetivo de analizar la relación entre los tipos sociométricos y las autoatribuciones académicas del fracaso se llevaron a cabo análisis de diferencias de medias para evaluar si existen diferencias entre estudiantes que presentan o no una categoría (ej., popular vs. no popular) y análisis de varianza (ANOVA) para evaluar las diferencias en atribuciones académicas inter-categorías. Para identificar entre qué categorías se encuentran las diferencias se llevaron a cabo comparaciones post-hoc (Prueba de Scheffé). Debido al elevado tamaño muestral del estudio, las pruebas $t$ de Student y la razón $F$ pueden detectar erróneamente diferencias estadísticamente significativas. Por esta razón se incluyó el índice $d$ (diferencia media tipificada) propuesto por Cohen (1988), que permite valorar la magnitud o el tamaño del efecto de las diferencias halladas. La interpretación del tamaño del efecto resulta sencilla: valores menores o iguales a 0.20 indican un tamaño del efecto muy pequeño o insignificante, entre 0.20 y 0.49 pequeño, entre 0.50 y 0.79 moderado y mayores de 0.80 un tamaño del efecto grande (Cohen, 1988).

El establecimiento de ecuaciones predictoras de los tipos sociométricos se realizó mediante la técnica estadística de regresión logística, siguiendo el procedimiento de regresión por pasos hacia delante basado en el estadístico de Wald, puesto que las variables evaluadas en el estudio son categóricas y no cumplen los supuestos del modelo lineal general. La $R^{2}$ de 
Nagelkerke permitió evaluar el ajuste de los modelos. En el análisis de regresión logística se presentan los coeficientes de cada variable en la ecuación de regresión y los estadísticos alcanzados por los modelos a la hora de clasificar a los sujetos según el grupo al que pertenecen (p. ej., popular, rechazado-agresivo, rechazado-tímido, olvidado, líder, simpático, colaborador, peleón, obediente y buen estudiante). El modelado logístico permite estimar la probabilidad de que ocurra un evento, suceso o resultado (p. ej., alta puntuación en autoatribución académica del fracaso a la (apacidad), frente a que no ocurra, en presencia de uno o más predictores (p. ej., tipo sociométrico rechazado-agresivo). Esta probabilidad es estimada mediante el estadístico Odd Ratio (OR), que se interpreta de la siguiente forma: OR > 1 indica que la probabilidad de que se dé el evento aumenta en presencia de esta variable, OR $<1$ indica que la probabilidad de que se dé el evento disminuye cuando la variable está presente, valores cercanos a 1 indican que esa variable ejerce escasa o nula influencia en la predicción del evento (De Maris, 2003).

\section{Resultados}

Tipos sociométricos, categorías conductuales y autoatribuciones académicas del fracaso

La Tabla 1 presenta las diferencias entre estudiantes según los tipos sociométricos y las categorías conductuales en relación a las autoatribuciones académicas del fracaso (Lectura, Matemáticas y General).

Tabla 1. Diferencias en las puntuaciones de autoatribución académica del fracaso en General de los estudiantes en función de los tipos sociométricos y categorías conductuales.

\begin{tabular}{|c|c|c|c|c|c|c|c|c|c|c|c|c|c|c|c|}
\hline \multirow[t]{2}{*}{$\begin{array}{l}\text { Tipo } \\
\text { sociométrico }\end{array}$} & \multicolumn{2}{|c|}{$\begin{array}{l}\text { Fracaso atri- } \\
\text { buido a la } \\
\text { capacidad }\end{array}$} & \multicolumn{3}{|c|}{$\begin{array}{c}\text { Significación } \\
\text { estadística y } \\
\text { magnitud } \\
\text { de las diferencias }\end{array}$} & \multicolumn{2}{|c|}{$\begin{array}{l}\text { Fracaso } \\
\text { atribuido al } \\
\text { esfuerzo }\end{array}$} & \multicolumn{3}{|c|}{$\begin{array}{c}\text { Significación } \\
\text { estadística y } \\
\text { magnitud } \\
\text { de las diferencias }\end{array}$} & \multicolumn{2}{|c|}{$\begin{array}{l}\text { Fracaso atri- } \\
\text { buido a causas } \\
\text { externas }\end{array}$} & \multicolumn{3}{|c|}{$\begin{array}{c}\text { Significación } \\
\text { estadística y } \\
\text { magnitud } \\
\text { de las diferencias }\end{array}$} \\
\hline & M & (DT) & $\mathrm{t}$ & $\mathrm{p}$ & d & M & (DT) & $\mathrm{t}$ & $\mathrm{p}$ & d & M & (DT) & $\mathrm{t}$ & $\mathrm{p}$ & d \\
\hline No Popular & 4.80 & 1.47 & \multirow{2}{*}{.03} & \multirow{2}{*}{.97} & \multirow{2}{*}{ - } & 5.62 & 1.22 & \multirow{2}{*}{.61} & \multirow{2}{*}{.54} & & 6.48 & 1.16 & \multirow{2}{*}{.84} & \multirow{2}{*}{.39} & \multirow{2}{*}{-} \\
\hline Popular & 4.79 & 1.44 & & & & 5.56 & 1.31 & & & & 6.40 & 1.05 & & & \\
\hline No Rechazado-agresivo & 4.78 & 1.46 & \multirow{2}{*}{-2.23} & \multirow{2}{*}{.02} & \multirow{2}{*}{-0.48} & 5.60 & 1.23 & \multirow{2}{*}{-1.96} & \multirow{2}{*}{.05} & \multirow{2}{*}{-0.34} & 6.48 & 1.14 & \multirow{2}{*}{2.94} & \multirow{2}{*}{.00} & \multirow{2}{*}{0.51} \\
\hline Rechazado-agresivo & 5.34 & 1.50 & & & & 6.02 & 1.17 & & & & 5.90 & 1.35 & & & \\
\hline No Rechazado-tímido & 4.78 & 1.46 & \multirow{2}{*}{-3.24} & \multirow{2}{*}{.00} & \multirow{2}{*}{-0.70} & 5.60 & 1.23 & \multirow{2}{*}{-2.03} & \multirow{2}{*}{.04} & \multirow{2}{*}{-0.45} & 6.47 & 1.14 & \multirow{2}{*}{1.44} & \multirow{2}{*}{.15} & \multirow{2}{*}{-} \\
\hline Rechazado-tímido & 5.80 & 1.62 & & & & 6.15 & 1.39 & & & & 6.12 & 1.39 & & & \\
\hline
\end{tabular}




\begin{tabular}{|c|c|c|c|c|c|c|c|c|c|c|c|c|c|c|c|}
\hline No Olvidado & 4.78 & 1.47 & \multirow{2}{*}{-1.21} & \multirow{2}{*}{.22} & & 5.61 & 1.24 & \multirow{2}{*}{-.75} & \multirow{2}{*}{.45} & & 6.47 & 1.14 & \multirow{2}{*}{1.19} & \multirow{2}{*}{.23} & \multirow{2}{*}{-} \\
\hline Olvidado & 5.01 & 1.31 & & & & 5.72 & 1.12 & & & & 6.30 & 1.27 & & & \\
\hline No Líder & 4.85 & 1.45 & \multirow{2}{*}{2.95} & \multirow{2}{*}{.00} & \multirow{2}{*}{0.20} & 5.59 & 1.22 & \multirow{2}{*}{-.89} & \multirow{2}{*}{.37} & & 6.47 & 1.15 & \multirow{2}{*}{.14} & \multirow{2}{*}{.89} & \multirow{2}{*}{-} \\
\hline Líder & 4.56 & 1.50 & & & & 5.67 & 1.32 & & & & 6.46 & 1.15 & & & \\
\hline No Simpático & 4.75 & 1.43 & \multirow{2}{*}{-.72} & \multirow{2}{*}{.46} & \multirow{2}{*}{ - } & 5.58 & 1.21 & \multirow{2}{*}{-1.46} & \multirow{2}{*}{.14} & & 6.45 & 1.15 & \multirow{2}{*}{-.92} & \multirow{2}{*}{.35} & \multirow{2}{*}{-} \\
\hline Simpático & 4.82 & 1.57 & & & & 5.69 & 1.35 & & & & 6.52 & 1.12 & & & \\
\hline No Colaborador & 4.95 & 1.46 & \multirow{2}{*}{7.54} & \multirow{2}{*}{.00} & \multirow{2}{*}{0.49} & 5.68 & 1.24 & \multirow{2}{*}{4.31} & \multirow{2}{*}{.00} & \multirow{2}{*}{0.28} & 6.48 & 1.17 & \multirow{2}{*}{.55} & 58 & 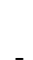 \\
\hline Colaborador & 4.25 & 1.34 & & & & 5.34 & 1.22 & & & & 6.44 & 1.09 & & .50 & 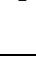 \\
\hline No Peleón & 4.73 & 1.44 & 151 & 12 & & 5.57 & 1.24 & 200 & 04 & 011 & 6.46 & 1.13 & & 72 & \\
\hline Peleón & 4.88 & 1.51 & -1.04 & .12 & & 5.74 & 1.23 & -2.05 & .04 & -0.14 & 6.48 & 1.22 & 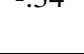 & 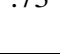 & \\
\hline No Obediente & 4.78 & 1.48 & 04 & 06 & & 5.63 & 1.23 & 80 & 27 & & 6.46 & 1.12 & & 80 & \\
\hline Obediente & 4.78 & 1.43 & -.04 & .90 & - & 5.56 & 1.31 & .89 & .31 & - & 6.48 & 1.21 & -.25 & .80 & - \\
\hline No Buen estudiante & 4.97 & 1.47 & 841 & 00 & 055 & 5.72 & 1.23 & 512 & 00 & 033 & 6.47 & 1.17 & -16 & 86 & - \\
\hline Buen estudiante & 4.19 & 1.28 & 0.41 & .00 & 0.0 & 5.31 & 1.22 & 5.12 & .00 & 0.3 & 6.48 & 1.09 & -.10 & .00 & \\
\hline
\end{tabular}

Los resultados indican que se encuentran puntuaciones medias significativamente más altas en el grupo de estudiantes rechazados-agresivos frente a los no rechazados-agresivos, en el grupo de rechazados-tímidos frente a los no rechazados-tímidos, en el grupo de no líderes frente a líderes, en el grupo de no colaboradores frente a colaboradores y en el grupo de no buenos estudiantes frente a los nominados como buenos estudiantes en cuanto a la autoatribución del fracaso en lectura a la capacidad. El tamaño del efecto de estas diferencias es de pequeña magnitud en todos los casos $(d<0.50)$, excepto en el grupo de rechazados-tímidos, cuyo tamaño del efecto es de magnitud moderada $(d \geq 0.50)$. En cuanto a la autoatribución del fracaso en lectura al esfuerzo, se encuentran puntuaciones medias significativamente más altas en el grupo de estudiantes no colaboradores frente a los colaboradores, en el grupo de peleones frente a los no peleones y en el grupo de no buenos estudiantes frente a los nominados como buenos estudiantes. El tamaño del efecto de estas diferencias es de pequeña magnitud en todos los casos $(d<0.50)$. Respecto a la autoatribución del fracaso en lectura a causas externas, presenta puntuaciones medias significativamente más altas únicamente el grupo de no rechazados-agresivos frente a los rechazados-agresivos, siendo el tamaño del efecto de esta diferencia de pequeña magnitud $(d<0.50)$.

Por otra parte, se encuentran puntuaciones medias significativamente más altas en el grupo de estudiantes rechazados-tímidos frente a los no rechazados-tímidos, en el grupo de no 
líderes frente a los líderes, en el grupo de no colaboradores frente a los colaboradores y en el grupo de no buenos estudiantes frente a los nominados como buenos estudiantes, en la autoatribución del fracaso en matemáticas a la capacidad. El tamaño del efecto de estas diferencias es de pequeña magnitud en el caso de los estudiantes líderes y colaboradores $(d<0.50)$, y de magnitud moderada en el grupo de rechazados-tímidos y buenos estudiantes $(d \geq 0.50)$. En cuanto a la autoatribución del fracaso en matemáticas al esfuerzo, se encuentran puntuaciones medias significativamente más altas en el grupo de rechazados-tímidos frente a los no rechazados-tímidos, en el grupo de simpáticos frente a los no-simpáticos, en el grupo de no colaboradores frente a los colaboradores y en el grupo de no buenos estudiantes frente a los nominados como buenos estudiantes. El tamaño del efecto de estas diferencias es de pequeña magnitud en todos los casos $(d<0.50)$. Respecto a la autoatribución del fracaso en matemáticas a causas externas, se encuentran puntuaciones medias significativamente más altas únicamente en el grupo de no rechazados-agresivos frente a los rechazados agresivos, siendo el tamaño del efecto de esta diferencia de pequeña magnitud $(d<0.50)$.

Finalmente, se encuentran puntuaciones medias significativamente más altas en el grupo de estudiantes rechazados-agresivos frente a no rechazados-agresivos, en el grupo de rechazados-tímidos frente a no rechazados-tímidos, en el grupo de no líderes frente a líderes, en el grupo de no colaboradores frente a colaboradores y en el grupo de no buenos estudiantes frente a los nominados como buenos estudiantes, en la autoatribución del fracaso en general a la capacidad. El tamaño del efecto de estas diferencias es de pequeña magnitud en el grupo de rechazados-agresivos, no líderes y no colaboradores $(d<0.50)$, y de magnitud moderada $(d$ $\geq 0.50$ ) en el grupo de rechazados-tímidos y no buenos estudiantes. En cuanto a la autoatribución del fracaso en general al esfuerzo, se encuentran puntuaciones medias significativamente más altas en el grupo de estudiantes rechazados-agresivos frente a no rechazados-agresivos, en el grupo de rechazados-tímidos frente a no rechazados-tímidos, en el grupo de no colaboradores frente a colaboradores, en el grupo de peleones frente a no peleones y en el grupo de no buenos estudiantes frente a los nominados como buenos estudiantes. El tamaño del efecto de estas diferencias es de pequeña magnitud en todos los casos $(d<0.50)$. Respecto a la autoatribución del fracaso en general a causas externas, se encuentran puntuaciones medias significativamente más altas únicamente en el grupo de estudiantes no rechazados-agresivos frente a rechazados-agresivos. El tamaño del efecto de esta diferencia es de magnitud mode$\operatorname{rada}(d \geq 0.50)$. 
El análisis de varianza (ANOVA) muestra que el grupo de estudiantes rechazadostímidos presenta puntuaciones medias significativamente más altas que los populares $(p<.05)$ en la autoatribución del fracaso en lectura a la capacidad, siendo el tamaño del efecto de esta diferencia de magnitud moderada $(d \geq 0.50)$. Los estudiantes simpáticos presentan puntuaciones significativamente más altas que los estudiantes colaboradores $(p<.05)$ en la autoatribución del fracaso en lectura a la capacidad, siendo el tamaño del efecto de esta diferencia de gran magnitud $(d \geq 0.80)$. El grupo de estudiantes rechazados-tímidos presenta puntuaciones medias significativamente más altas que los estudiantes populares $(p<.05)$ en la autoatribución del fracaso en matemáticas al esfuerzo, siendo el tamaño del efecto de esta diferencia de magnitud moderada $(d \geq 0.50)$. Asimismo, los estudiantes simpáticos presentan puntuaciones significativamente más altas que los estudiantes colaboradores y buenos estudiantes $(p<.05)$, siendo el tamaño del efecto de estas diferencias de magnitud moderada ( $d \geq 0.50)$. Por otra parte, los estudiantes rechazados-tímidos presentan puntuaciones significativamente más altas que los estudiantes populares $(p<.05)$ en la autoatribución del fracaso en general a la capacidad, siendo el tamaño del efecto de esta diferencia de magnitud moderada $(d \geq 0.50)$. Así mismo, los estudiantes simpáticos presentan puntuaciones significativamente más altas que los estudiantes colaboradores $(p<.05)$, siendo el tamaño del efecto de esta diferencia de gran magnitud $(d \geq 0.80)$. Respecto a la autoatribución del fracaso en general al esfuerzo, los estudiantes populares presentan puntuaciones significativamente más bajas que los estudiantes simpáticos $(p<.05)$, siendo el tamaño del efecto de esta diferencia de magnitud moderada ( $d$ $\geq 0.50$ ). Por último, en cuanto a la autoatribución del fracaso en general a causas externas, los estudiantes peleones presentan puntuaciones significativamente más altas que los estudiantes colaboradores $(p<.05)$, siendo el tamaño del efecto de esta diferencia de magnitud moderada $(d \geq 0.50)$.

Predicción de las autoatribuciones académicas del fracaso en función de los tipos sociométricos y categorías conductuales

Los análisis de regresión logística binaria mostraron que los tipos sociométricos y las categorías conductuales resultaron una variable estadísticamente significativa para la predicción de la autoatribución académica del fracaso. 
A partir de la muestra analizada fue posible crear ocho modelos logísticos que pronosticaron la probabilidad de autoatribuir el fracaso en Lectura, Matemáticas y en General a la Capacidad, al Esfuerzo y a Causas Externas a través de los tipos sociométricos y categorías conductuales. Así, los tipos sociométricos y categorías conductuales las aptitudes intelectuales (popular, rechazado-agresivo, rechazado-tímido, olvidados, líder, simpático, colaborador, peleón, obediente y buen estudiante) fueron incluidas como variables predictoras en todos los modelos logísticos creados aunque no todas resultaron significativas.

La proporción de casos clasificados correctamente por los modelos logísticos osciló según el tipo de atribución académica del éxito analizado. El modelo de autoautoatribución de los fracasos en lectura a la capacidad permite una estimación correcta del $52 \%$ de los casos $\left(\mathrm{R}^{2}\right.$ Nagelkerke $\left.=.004\right)$ para los estudiantes rechazados-agresivos, un $53.1 \%$ en el grupo de líderes $\left(\mathrm{R}^{2}\right.$ Nagelkerke $\left.=.01\right)$, un $54.5 \%$ en el grupo de colaboradores $\left(\mathrm{R}^{2}\right.$ Nagelkerke $=$ $.02)$ y un $54.1 \%$ en el grupo de buenos estudiantes $\left(\mathrm{R}^{2}\right.$ Nagelkerke $\left.=.02\right)$. El modelo de autoautoatribución de los fracasos en lectura al esfuerzo permite una estimación correcta del $51.9 \%$ de los casos $\left(\mathrm{R}^{2}\right.$ Nagelkerke $\left.=.01\right)$ para los estudiantes colaboradores, un $53.1 \%$ en el grupo de peleones $\left(\mathrm{R}^{2}\right.$ Nagelkerke $\left.=.01\right)$ y un $52.8 \%$ en el grupo de buenos estudiantes $\left(\mathrm{R}^{2}\right.$ Nagelkerke =.01). El modelo de autoatribución de los fracasos en lectura a causas externas permite una estimación correcta del $56.1 \%$ de los casos $\left(\mathrm{R}^{2}\right.$ Nagelkerke $\left.=.01\right)$ para los estudiantes rechazados-agresivos y un $55 \%$ en el grupo de peleones $\left(\mathrm{R}^{2}\right.$ Nagelkerke $\left.=.002\right)$. El modelo de autoatribución de los fracasos en matemáticas a la capacidad permite una estimación correcta del $54.8 \%$ de los casos $\left(\mathrm{R}^{2}\right.$ Nagelkerke $\left.=.02\right)$ para los estudiantes colaboradores y un $56.2 \%$ en el grupo de buenos estudiantes $\left(\mathrm{R}^{2}\right.$ Nagelkerke $\left.=.03\right)$. El modelo de autoatribución de los fracasos en matemáticas al esfuerzo permite una estimación correcta del $54.6 \%$ de los casos $\left(\mathrm{R}^{2}\right.$ Nagelkerke $\left.=.02\right)$ para los estudiantes colaboradores y un $54.5 \%$ en el grupo de buenos estudiantes $\left(\mathrm{R}^{2}\right.$ Nagelkerke $\left.=.02\right)$. El modelo de autoatribución de los fracasos en matemáticas a causas externas permite una estimación correcta del $54.4 \%$ de los casos $\left(\mathrm{R}^{2}\right.$ Nagelkerke $=.01)$ para los estudiantes populares. El modelo de autoatribución de los fracasos en general a la capacidad permite una estimación correcta del $52.2 \%$ de los casos $\left(\mathrm{R}^{2}\right.$ Nagelkerke $=.01)$ para los estudiantes olvidados, un $53.7 \%$ en el grupo de líderes $\left(\mathrm{R}^{2}\right.$ Nagelkerke $=$ $.01)$, un $59.1 \%$ en el grupo de colaboradores $\left(\mathrm{R}^{2}\right.$ Nagelkerke $\left.=.05\right)$, un $51.7 \%$ en el grupo de peleones $\left(\mathrm{R}^{2}\right.$ Nagelkerke $\left.=.004\right)$ y un $60.3 \%$ en el grupo de buenos estudiantes $\left(\mathrm{R}^{2}\right.$ Nagelkerke $=.06)$. El modelo de autoatribución de los fracasos en general al esfuerzo permite una 
estimación correcta del $55.6 \%$ de los casos $\left(\mathrm{R}^{2}\right.$ Nagelkerke $\left.=.02\right)$ para los estudiantes colaboradores, un $52.6 \%$ en el grupo de peleones $\left(\mathrm{R}^{2}\right.$ Nagelkerke $\left.=.01\right)$ y un $56.2 \%$ en el grupo de buenos estudiantes $\left(\mathrm{R}^{2}\right.$ Nagelkerke $\left.=.02\right)$. El modelo de autoatribución de los fracasos en general a causas externas permite una estimación correcta del $52.6 \%$ de los casos $\left(\mathrm{R}^{2}\right.$ Nagelkerke $=.004)$ para los estudiantes rechazados-agresivos

Las OR de los modelos logísticos para la predicción de las autoatribuciones académicas del fracaso muestran (véase Tabla 2): a) que los estudiantes rechazados-agresivos presentan un $105 \%$ más de probabilidad de autoatribuir sus fracasos en lectura a la capaci$d a d$, los líderes un 32\% menos de probabilidad, los colaboradores un $43 \%$ menos de probabilidad y los nominados como buenos estudiantes un $40 \%$ menos de probabilidad; b) que los estudiantes colaboradores presentan un $25 \%$ menos de probabilidad de autoatribuir sus fracasos en lectura al esfuerzo, los peleones un $32 \%$ más de probabilidad y los buenos estudiantes un $30 \%$ menos de probabilidad; c) que los estudiantes rechazados-agresivos presentan un $68 \%$ menos de probabilidad de autoatribuir sus fracasos en lectura a causas externas y los peleones un $14 \%$ menos de probabilidad; c) que los estudiantes colaboradores presentan un $43 \%$ menos de probabilidad de autoatribuir sus fracasos en matemáticas a la capacidad y los buenos estudiantes un 52\% menos de probabilidad; d) que los estudiantes colaboradores presentan un $44 \%$ menos de probabilidad de autoatribuir sus fracasos en matemáticas al esfuerzo y los buenos estudiantes un $43 \%$ menos de probabilidad; e) que los estudiantes populares presentan un $28 \%$ menos de probabilidad de autoatribuir sus fracasos en matemáticas a causas externas; f) que los estudiantes olvidados presentan un $89 \%$ más de probabilidad de autoatribuir sus fracasos en general a la capacidad, los líderes un $28 \%$ menos de probabilidad, los colaboradores un $59 \%$ menos de probabilidad, los peleones un $30 \%$ más de probabilidad y los buenos estudiantes un 65\% menos de probabilidad; g) que los estudiantes colaboradores presentan un $42 \%$ menos de probabilidad de autoatribuir sus fracasos en general al esfuerzo, los peleones un $41 \%$ más de probabilidad y los buenos estudiantes un $44 \%$ menos de probabilidad; y h) que los estudiantes rechazados-agresivos presentan un 52\% menos de probabilidad de autoatribuir sus fracasos en general a causas externas.

Tabla 2. Resultados derivados de la regresión logística binaria para la probabilidad de presentar altas puntuaciones de autoatribución causal del fracaso en general en función de los tipos sociométricos y categorías conductuales 


\begin{tabular}{|c|c|c|c|c|c|c|c|}
\hline$V D$ & $V I$ & $B$ & E.T. & Wald & $p$ & $O R$ & I.C. $95 \%$ \\
\hline \multicolumn{8}{|c|}{ Atribuciones causales } \\
\hline \multirow{10}{*}{ Fracaso Capacidad } & Olvidado & .64 & .26 & 5.93 & .01 & 1.89 & $1.13-3.16$ \\
\hline & Constante & .06 & .06 & 1.01 & .31 & 1.06 & \\
\hline & Lider & -.33 & .13 & 6.36 & .01 & .72 & $.56-.93$ \\
\hline & Constante & .13 & .07 & 3.73 & .05 & 1.14 & \\
\hline & Colaborador & -.89 & .13 & 43.44 & .00 & .41 & $.31-.53$ \\
\hline & Constante & .28 & .07 & 17.76 & .00 & 1.33 & \\
\hline & Peleón & .26 & .13 & 3.85 & .05 & 1.30 & $1.00-1.68$ \\
\hline & Constante & -.01 & .07 & .01 & .92 & .99 & \\
\hline & Buen estudiante & -1.05 & .14 & 58.11 & .00 & .35 & $.27-.46$ \\
\hline & Constante & .31 & .07 & 22.02 & .00 & 1.37 & \\
\hline \multirow{6}{*}{ Fracaso Esfuerzo } & Colaborador & -.55 & .13 & 17.36 & .00 & .58 & $.44-.75$ \\
\hline & Constante & .17 & .07 & 6.49 & .01 & 1.19 & \\
\hline & Peleón & .34 & .13 & 6.70 & .01 & 1.41 & $1.09-1.83$ \\
\hline & Constante & -.04 & .07 & .32 & .57 & .96 & \\
\hline & Buen estudiante & -.59 & .13 & 19.57 & .00 & .56 & $.43-.72$ \\
\hline & Constante & .21 & .07 & 9.76 & .00 & 1.23 & \\
\hline & Rechazado-agresivo & -.74 & .36 & 4.24 & .04 & .48 & $.23-.96$ \\
\hline Fracaso Externas & Constante & .09 & .05 & 2.74 & .09 & 1.09 & \\
\hline
\end{tabular}

\section{Discusión y Conclusiones}

El objetivo de este estudio fue analizar la relación entre los tipos sociométricos y las autoatribuciones académicas del fracaso en una muestra de adolescentes españoles. A diferencia de estudios anteriores, este trabajo amplía el número de tipos sociométricos examinados y las diferentes categorías conductuales que pueden aparecer dentro de un aula/grupo social. Además, y a diferencia también de trabajos previos, esta investigación ha contemplado dicha relación teniendo en cuenta el análisis de los tamaños del efecto, recomendado por distintos autores (e.g., Cohen, 1988; García, Ortega y De la Fuente, 2011) para determinar la magnitud de las diferencias encontradas, es decir, su significado teórico y práctico.

Los resultados de este estudio revelan que los tipos sociométricos y variables conductuales fueron variables predictoras significativas de las autoatribuciones académicas del fracaso ya que aquellos alumnos que recibieron mayor número de nominaciones negativas (estudiantes no populares, rechazados-agresivos, rechazados-tímidos, olvidados, no líderes, peleones, no colaboradores y no buenos estudiantes) por sus iguales presentaron puntuaciones más 
altas en la autoatribución académica del fracaso a la Capacidad y al Esfuerzo (en Lectura, Matemáticas y en General) que aquellos estudiantes nominados positivamente por sus compañeros (populares, no rechazados-agresivos, no rechazados-tímidos, no olvidados, líderes, no peleones, colaboradores y buenos estudiantes), aunque sólo resultaron estadísticamente significativas para los estudiantes nominados como rechazados-agresivos, rechazados-tímidos, no líderes, no colaboradores y no buenos estudiantes, confirmándose, de este modo, la primera hipótesis. Los resultados de esta investigación van en consonancia con los resultados de estudios previos en población extranjera y española que destacan la relación existente entre las atribuciones produce en el sujeto unos sentimientos de autoconfianza, lo que influye directamente en su autoconcepto y su propia competencia social y sobre la motivación de rendimiento y la motivación de aprendizaje (Hayamizu y Weiner, 1991; Jiménez, 2003; Muñoz, Trianes y Jiménez, 1994; Zhao y Su, 2005).

Adicionalmente, los análisis de varianza realizados revelaron diferencias estadísticamente significativas en algunos tipos sociométricos y categorías conductuales en función de las autoatribuciones académicas del fracaso. En este sentido, los estudiantes rechazadostímidos presentan puntuaciones significativamente más altas que los estudiantes populares en la autoatribución del fracaso en General a la Capacidad. Este resultado puede explicarse siguiendo a Wichmann, Coplan y Daniels (2004), que afirman que los estudiantes identificados por los compañeros como socialmente retraídos presentan un patrón atribucional de autorechazo (atribución interna y estable de los fracasos) y familiaridad con el fracaso social en las interacciones sociales, menor autoeficacia para desarrollar metas asertivas y de resolución de problemas, y una preferencia mayor hacia estrategias inhibidas y no asertivas para el manejo de situaciones conflictivas, confirmando cierta semejanza o paralelismo entre el contexto académico y social indicado por diversos autores (Weiner, 2004). De acuerdo a lo propuesto en la segunda hipótesis, los tipos sociométricos resultaron ser un predictor significativo de las autoatribuciones académicas, puesto que los estudiantes nominados negativamente por sus compañeros (como rechazados-agresivos, peleones y olvidados) presentaron mayor probabilidad de atribuir sus fracasos académicos a la Capacidad y al Esfuerzo. Además, la razón $F$ es muy sensible respecto al tamaño de la muestra reclutada, pudiendo detectar erróneamente diferencias estadísticamente significativas (Cohen, 1988). Con el fin de evitar este sesgo se calcularon los tamaños del efecto propuestos por Cohen (1988). Sin embargo, éstos confirmaron que la magnitud de las diferencias halladas fue moderada en la mayoría de los casos. 
Los resultados de este estudio ponen de relieve, por una parte, la utilidad de los métodos sociométricos en las investigaciones realizadas en centros de enseñanza, entre otros motivos, por su fácil aplicación y su validez empírica (Muñoz, Moreno y Jiménez, 2008) y, por otra parte, por la relación de los tipos sociométricos con diferentes variables cognitivomotivacionales (e.g., las autoatribuciones académicas) y su influencia sobre el ajuste social.

Esta investigación no está exenta de limitaciones. En primer lugar, aunque el muestreo empleado garantiza la representatividad de la muestra reclutada respecto a la población diana, los resultados hallados en esta investigación no pueden generalizarse a estudiantes españoles de otros niveles educativos (Educación Infantil, Primaria, Bachillerato y Educación Superior). Futuras investigaciones deberían confirmar si los resultados hallados en ESO difieren o se mantienen en otros niveles educativos. En segundo lugar, tampoco resultaría adecuado establecer generalizaciones de los hallazgos a estudiantes españoles de ESO diagnosticados con trastornos del aprendizaje o trastornos psicopatológicos, aspectos que pueden alterar claramente el comportamiento social y académico de los estudiantes. Además, teniendo en cuenta el principio de especificidad situacional que caracteriza el comportamiento social, difícilmente los resultados obtenidos podrían extrapolarse a estudiantes de Educación Secundaria de otras culturas y etnias. En tercer lugar, sería interesante que futuros trabajos incluyeran distintas fuentes de evaluación de la conducta social (autoinformes, profesores) para analizar la concordancia inter-fuentes. Además, futuras investigaciones deberían analizar los distintos grupos de estudiantes ignorados y rechazados (Estévez et al., 2006) así como las categorías sociométricas de controvertidos y medios, pues la omisión de estos análisis puede generar resultados incompletos a la hora de clasificar a los alumnos en un determinado tipo sociométrico. En quinto lugar, sería aconsejable que futuros trabajos utilicen diseños longitudinales a fin de aportar datos más concluyentes respecto a las relaciones de influencia entre estas variables. Finalmente, en el presente estudio se plantea conocer la capacidad predictiva de los tipos sociométricos y características conductuales sobre las autoatribuciones académicas y no al revés (capacidad predictiva de las atribuciones académicas sobre los tipos sociométricos y características conductuales). Aunque lo lógico es pensar es que exista un efecto recíproco, futuras investigación podrían analizar esta cuestión elaborando dos modelos de ecuaciones estructurales para ver que hipótesis es la más sostenible o, en cualquier caso, cuál es la fuerza de las asociaciones en ambos modelos. 
A nivel práctico, los resultados de esta investigación apuntan, en primer lugar, a trabajar con los estudiantes para identificar y tratar los problemas de aprendizaje que pueden interferir en el desempeño escolar. En este sentido, resultaría de gran importancia conocer cuál es el estilo atribucional de los estudiantes y orientarles para que sea lo más adaptativo posible, es decir, que atribuyan sus éxitos a causas estables, controlables e internas, como son la Capacidad y el Esfuerzo, con el objetivo de aumentar el desempeño académico y, al mismo tiempo, mejorar el ajuste social. En segundo lugar, es importante trabajar en la identificación concreta de factores de riesgo y de protección para el rendimiento escolar, acompañada de un entrenamiento adecuado que posibilite la puesta en marcha de estrategias cognitivas y de autorregulación eficaces que estimulen el desarrollo de sentimientos de confianza en las propias capacidades (González-Pienda et al., 2000), mejorando de ese modo el rendimiento académico (de la Fuente, Justicia, Sander y Cardelle-Elawar, 2014). La evidencia empírica muestra que la identificación y el manejo de estos factores en la escuela mejora el bienestar emocional del niño (Baker, 2008) y su capacidad de resiliencia (Suriá, 2016). Finalmente, es necesario destacar que, pese a que la importancia del estudio de los tipos sociométricos en los adolescentes radica principalmente en su efecto sobre el desarrollo social y las habilidades de interacción (Bukowski, Bredgen y Vitaro, 2007), los tipos sociométricos no sólo afectan al área social, sino también al ámbito académico (Meijs, Cillessen, Scholte, Segers y Spijkerman, 2010). Concretamente, diferentes estudios han señalado que el ser identificado como preferido en la adolescencia resulta una variable influyente en la repetición de curso (Lubbers et al., 2006), el rendimiento escolar (Véronneau, Vitaro, Brendgen, Dishion y Tremblay, 2010) y las expectativas sobre el desempeño académico (Cillessen y Mayeux, 2007). Estos aspectos hacen especialmente necesaria la consideración de las relaciones entre iguales en la adolescencia, ya que el comienzo de la educación Secundaria es uno de los factores influyentes en la mayor interacción con los iguales, pues la ambigüedad inicial que puede generar la nueva situación y el frecuente cambio de compañeros de clase hace necesario interactuar con un mayor número de estudiantes (Herrenkohl et al., 2001). 


\section{Referencias}

Bain, S. K. y Allin, J. D. (2005). Review of Stanford-Binet Intelligence Scales, Fifth Edition. Journal of Psychoeducational Assessment, 23, 87-95.

doi: $10.1177 / 073428290502300108$

Baker, J. A. (2008). Assessing school risk and protective factors. In B. Doll y J. A. Cummings (Eds.), Transforming school mental health services: Population-based approaches to promoting the competency and wellness of children (pp. 43-65). Thousand Oaks, CA: Corwin Press.

Bukowski, W. M., Brendgen, M. y Vitaro, F. (2007). Peers and socialization: Effects on externalizing and internalizing problems. En J. E. Grusec y P. D. Hasting (Eds.), Handbook of Socialization: Theory and research (pp. 355-381). Nueva York: Guilford Press.

Chen, X., Chang, L. y He, Y. (2003). The peer group as a context: mediating and moderating effects on relations between achievement and social functioning in Chinese children. Child Development, 74(3), 710-727.

doi: $\underline{10.1111 / 1467-8624.00564}$

Cillessen, A. H. N. y Mayeux, L. (2007). Expectations and perceptions at school transitions: the role of peer status and aggression. Journal of School Psychology, 45, 567-586. doi: 10.1016/j.jsp.2007.05.004

Cohen, J. (1988). Statistical power analysis for the behavioral sciences (2nd Ed.). Hillsdale, NJ: Erlbaum.

Coronel, C. P., Levin, M. y Mejail, S. (2011). Las habilidades sociales en adolescentes tempranos de diferentes contextos socioeconómicos. Electronic Journal of Research in Educational Psychology, 9(23), 241-261.

de la Fuente, J., Justicia, F., Sander, P. y Cardelle-Elawar, M. (2014). Autorregulación personal y enseñanza reguladora para predecir el rendimiento y la confianza académica: nueva evidencia para el modelo DIDEPRO. Electronic Journal of Research in Educational Psychology, 12(3), 597-620. doi: 10.14204/ejrep.34.14031

De Maris, A. (2003). Logistic regression. In J. A. Schinka y W. F. Velicer (Eds.), Research methods in psychology (pp. 509-532). Nueva Jersey: John Wiley \& Sons.

Diaz-Aguado, M. (2005). La violencia entre iguales en la adolescencia y su prevención desde la escuela. Psicothema, 17(4), 549-558. 
Estévez, E., Herrero, J., Martínez, B. y Musitu, G. (2006). Aggressive and nonaggressive rejected students: an analysis of their differences. Psychology in the Schools, 43, 387-400. doi: $10.1002 /$ pits.20152

Garaigordobil, M. (2006). Psychopathological symptoms, social skills and personality traits: A study with adolescents. The Spanish Journal of Psychology, 9, 182-192. doi: $\underline{10.1017 / \mathrm{s} 1138741600006089}$

García, J., Ortega, E. y De la Fuente, L. (2011). The use of the effect size in JCR Spanish Journal of Psychology: From theory to Fac. The Spanish Journal of Psychology, 14, 1050-1055. doi: 10.5209/rev_SJOP.2011.v14.n2.49

García-Bacete, F. J. (2007). La identificación de los alumnos rechazados, preferidos, ignorados y controvertidos en el aula. Revista de Psicología General y Aplicada, 60, 25-46.

González, J. (1990). Sociometría por ordenador. Valencia: Conselleria de Cultura, Educació i Ciencia.

González-Pienda, J. A., Núñez, J. C., González-Pumariega, S., Álvarez, L., Roces, C., García, M., González, P., Cabanach, R. G. y Valle, A. (2000). Autoconcepto, proceso de atribución causal y metas académicas en niños con y sin dificultades de aprendizaje. Psicothema, 12(4), 548-556.

Hayamizu, T. y Weiner, B. (1991). A test of Dweck's model of achievement goals are related to perceptions of ability. Journal of Experimental Education, 59, 226-234. doi: $\underline{10.1080 / 00220973.1991 .10806562}$

Herrenkohl, T. I, Guo, J., Kosterman, R., Hawkins, J.D., Catalano, R. F. y Smith, B. H. (2001). Early adolescent predictors of youth violence as mediators of childhood risks. Journal of Early Adolescence, 21, 447-469. doi: $10.1177 / 0272431601021004004$

Inglés, C. J., Delgado, B., García-Fernández, J. M., Ruiz-Esteban, C. y Díaz-Herrero, A. (2010). Sociometric types and social interaction styles in a sample of Spanish adolescents. The Spanish Journal of Psychology, 13, 728-738.

doi: $\underline{10.1017 / \mathrm{S} 1138741600002390}$

Inglés, C. J., Rodríguez-Marín, J. y González-Pienda, J. A. (2008). Adaptación de la Sydney Attribution Scale en población universitaria española. Psicothema, 20, 166-173.

Jiménez, M. (2003). Características emocionales y comportamentales de los grupos sociométricos desde una perspectiva múltiple. Psicología Conductual, 11(1), 41- 60. 
Lubbers, M. J., Van Der Werf, M. P. C., Snijders, T. A. B., Bert P. M., Creemers, B. P. M. y Kuyper, H. (2006). The impact of peer relations on academic progress in junior high. Journal of School Psychology, 44(6), 491-512. doi: $10.1016 /$ j.jsp.2006.07.005

Marsh, H. W. (1984). Relations among dimensions of self-attribution, dimensions of selfconcept and academic achievements. Journal of Educational Psychology, 76, 12911308. doi: $\underline{10.1037 / / 0022-0663.76 .6 .1291}$

Martín, E. (2011). The influence of diverse interaction contexts on students' sociometric status. The Spanish Journal of Psychology, 14, 88-98.

doi: $10.5209 /$ rev_SJOP.2011.v14.n1.7

Martínez-Arias, R., Martín, J. y Díaz-Aguado, M. J. (2009). Los métodos sociométricos en la psicología del desarrollo y educativa. Madrid: FOCAD. Disponible en web: http://www.cop.es/focad/pdf/010-FOCAD-02.pdf

Meijs, N., Cillessen, A. H. N., Scholte, R. H. J., Segers, E., y Spijkerman, R. (2010). Social intelligence and academic achievement as predictors of adolescent popularity. Journal of Youth and Adolescence, 39, 62-72. doi:10.1007/s10964-008-9373-9

Moreno, J. L. (1934). Who shall survive? A New Approach to the Problem of Human Relations. Washington, DC: Nervous and Mental Disease Publishing Co.

Muñoz Sánchez, A., Trianes Torres, M. V. y Jiménez Hernández, M. (1994). Una propuesta de educación de la competencia para las relaciones interpersonales a través del nuevo currículum educativo. Infancia y sociedad, 24, 47-77.

Muñoz Tinoco, V., Moreno Rodríguez, M. C. y Jiménez Lagares, I. (2008). Las tipologías de estatus sociométrico durante la adolescencia: contraste de distintas técnicas y fórmulas para su cálculo. Psicothema, 20, 665-671.

Núñez, J. C. y González-Pienda, J. A. (1994). Determinantes del rendimiento académico. Oviedo: Servicio de Publicaciones de la Universidad de Oviedo.

Pan, J. (2002). An attributional study of peer acceptance and rejection among middle school students. Psychological Science, 25(1), 64-68.

Peery, J. C. (1979). Popular, amiable, isolated, rejected: A reconceptualization of sociometric status in preschool children. Child Development, 50, 1231-1234. doi: $\underline{10.2307 / 1129356}$

Rubin, K. H., Bukowski, W. M. y Parker, J. G. (2006). Peer interactions, relationships and groups. En N. Eisenberg, W. Damon y R. M. Lerner (Eds.), Handbook of Child Psy- 
chology: Vol. 3. Social, emotional, and personality development (6th ed., pp. 571-645). New York: Wiley.

Suriá, R. (2016). Relación entre autoconcepto y perfiles de resiliencia en jóvenes con discapacidad. Electronic Journal of Research in Educational Psychology, 14(3), 450-473. doi: 10.14204/ejrep.40.15150

Veronneau, M. H., Vitaro, F., Brendgen, M., Dishion, T. J. y Tremblay, R. E. (2010). Transactional analysis of the reciprocal links between peer experiences and academic achievement from middle childhood to early adolescence. Developmental Psychology, 46, 773-790. doi: $\underline{10.1037 / a 0019816}$

Weiner, B. (1986). An attributional theory of motivation and emotion. New York: SpringerVerlag.

Weiner, B. (2004). Attribution theory revisited: transforming cultural plurality into theoretical unity. En D. M. McInerney y S. Van Etten (Eds.), Big theories revisited (pp. 13-29). Grenwich, CT.: Information Age Publishing.

Wichmann, C., Coplan, R. J. y Daniels, T. (2004). The social cognitions of socially withdrawn children. Social Development, 13, 377-392. doi: 10.1111/j.1467-9507.2004.00273.x

Zhao, Hong-Mei y Su, Yan-Jie. (2005). Peer Acceptance and Interpretation of Peer Rejection in Adolescence. Chinese Mental Health Journal, 19(5), 347-349. 